\title{
ESCISIÓN DE LA ZONA DE TRANSFORMACIÓN EN LESIONES DE CÉRVIX: ANÁLISIS DE 1.141 CASOS
}

\author{
Jorge Cabrera D. ${ }^{1}$, Juan Villanueva G. ${ }^{1}$, Fernando Heredia M. ${ }^{1}$, Lionel Correa S. ${ }^{1}$, \\ Juan Landeros S. ${ }^{1}$, Juanita Navarrete V. ${ }^{1}$, Francisco Mucientes H. ${ }^{2}$, Rodrigo Klaas- \\ sen P. ${ }^{2}$, Clemencia Cabrera F. ${ }^{a}$, Margarita Burgos G. ${ }^{a}$, Ivonne Riquelme E. a , Maritza \\ Flores $0^{3}$ \\ ${ }^{1}$ Departamento y Servicio de Obstetricia y Ginecología, ${ }^{2}$ Departamento de Patología, ${ }^{3}$ Departamento de Salud Pública, \\ Universidad de Concepción, Hospital Guillermo Grant B., Concepción.
}

a Matronas

\section{RESUMEN}

Objetivo: Analizar los resultados de la escisión de la zona de transformación del cérvix efectuadas en la Unidad de Patología Cervical del Hospital Guillermo Grant de Concepción. Método: Estudio descriptivo, retrospectivo, de los antecedentes clínicos e histológicos de 1.141 casos de escisión electro quirúrgica efectuados entre 1999 a 2005, con seguimiento mínimo de 18 meses. Resultados: De las 3.004 mujeres derivadas por citología positiva para neoplasia y/o HPV, a $1.141(37,9 \%)$ se les realizó escisión de la zona de transformación; 1.113 con asa electroquirúrgica $(97,5 \%)$ y $28(2,5 \%)$ cono frío con bisturí. Las lesiones intraepiteliales (LIE) de alto grado fueron las más frecuentes $(66,7 \%)$, seguidas por las LIE de bajo grado (7,8\%); el 6,5\% de las pacientes presentan etapas iniciales de carcinoma invasor; $33 \%$ de las piezas histológicas presentaron bordes comprometidos; $18,4 \%$ no presentaron lesión histológica, que sumadas a 7,8\% de LIE de bajo grado representan un $26,2 \%$ de mujeres consideradas como sobretratadas. El porcentaje a 18 meses de seguimiento en mujeres con LIE de alto grado fue de $85,5 \%$. Necesitaron de una segunda intervención 194 pacientes (17\%), 110 (9,6\%) por persistencia y recidivas de la lesión. Conclusión: Los resultados presentados son satisfactorios en el porcentaje de curación de las LIE de alto grado como en el aporte al diagnóstico precoz de carcinomas del cérvix y similares a estudios publicados tanto nacionales como extranjeros.

\section{PALABRAS CLAVES: Cáncer cérvicouterino, neoplasia intraepitelial, zona de transformación, coni- zación, asa electroquirúrgica}

\section{SUMMARY}

Objective: To analyze the results of 1,141 cervical excisions. Method: Descriptive and retrospective study of the clinical charts and biopsies of 1,141 cases with surgical resection of the uterine cervix performed from 1999 to 2005. Only patients with a minimum of 18 months of follow up were included. Results: From 3,004 patients with a positive Papanicolaou smear for neoplastic cells and/or condyloma, 1,113 (97.5\%) were treated with loop electrosurgical excisional procedure (LEEP) and $28(2.5 \%)$ with cold-knife conization. The most frequent lesion $(66.7 \%)$ found was high grade squamous intraepithelial lesions (SIL), follow by low grade SIL (7.8\%); 74 patients (6.5\%) presented with early stage of squamous cell carcinoma and $377(33 \%)$ of the histological specimens revealed positive margins; $210(18.4 \%)$ of the cases showed no histological lesion. 
There were a $26.2 \%$ of females that were considered to receive over treatment. After 18 months follow up $85.5 \%$ of patient were free of high grade SIL. A second surgical treatment was done in 194 patients (17\%) including 110 cases $(9.6 \%)$ with persistent or recurrence of the lesion. Conclusions: The results are satisfactory related to treatment of high grade SIL and it contributes to the early diagnosis of cervical cancer. The results are similar to other national and international series previously published.

\section{KEY WORDS: Cervix cancer, intraepithelial neoplasia, transformation zone, conisation, LEEP}

\section{INTRODUCCIÓN}

La tasa de mortalidad por cáncer cérvico-uterino en Chile ha tenido una tendencia sostenida a disminuir desde hace varios años. El año 1990 había una tasa de 11,8 por 100.000 mujeres y 8,2 por 100.000 mujeres en el último reporte del año 2005, constituyéndose actualmente en la quinta causa de muerte de la mujer, que se manifiesta a través del fallecimiento aproximado de dos mujeres por día $(1,2,3)$.

La mortalidad en la $8^{\text {a }}$ Región se ha mantenido con una tasa superior al promedio nacional, sin una tendencia clara a disminuir. En el año 2002 hubo 81 defunciones que representan la tasa más baja (8,3 por 100.000 mujeres) y el año 1993 la más alta (17,6 por 100.000 mujeres). En el último reporte del año 2005 la mortalidad fue de 10,9 por 100.000 mujeres. Estas oscilaciones se repiten en casi todas las regiones del país (1).

La pesquisa mediante la citología de Papanicolaou y el tratamiento consecutivo de las lesiones preinvasoras aseguran una alta curación, e impiden el desarrollo a cáncer invasor, son pilares fundamentales del enfrentamiento y manejo de esta patología (4).

El advenimiento del asa electroquirúrgica ha sido un aporte trascendente en el manejo de las lesiones preclínicas. Su utilización produjo un aumento notable en el número de procedimientos, por ser ambulatorios, con buena aceptación de las mujeres, escasas complicaciones y bajo costo $(5,6)$.

El objetivo de esta comunicación es analizar los resultados de 1.141 estudios histológicos obtenidos mediante la escisión de la zona de transformación del cuello uterino, realizadas mayoritariamente con asa electroquirúrgica y que constituye la mayor experiencia nacional publicada a la fecha.

\section{MATERIAL Y MÉTODO}

Entre los años 1999 y 2005 se derivaron a la Unidad de Patología Cervical (UPC) para estudio del cérvix 3.004 mujeres con citología positiva para neoplasia y/o sospecha de condiloma (HPV). De este grupo se realizaron $1.141(37,9 \%)$ escisiones de la zona de transformación, que corresponde al universo de este análisis. Se efectuó seguimiento por un lapso mínimo de 18 meses.

La UPC, es atendida por 6 médicos ginecólogos y 3 matronas. El estudio consistió en realizar colposcopia, repetir la citología, realizar biopsias dirigidas e indicar el procedimiento quirúrgico en forma diferida.

La escisión de la zona de transformación con asa electroquirúrgica se realizó en forma ambulatoria en la mayoría de los casos, previa anestesia local, mediante infiltración de lidocaína en 2 puntos del cérvix ( 3 y 9 horarias) obteniendo fragmentos correspondientes a labio anterior, posterior y canal endocervical. Seguidamente, fueron sometidas a electrocauterización del borde y lecho. Eventualmente se aplicó subgalato de bismuto al lecho, al grupo de pacientes con hemorragia post procedimiento. Los conos fríos se realizaron con bisturí en pacientes hospitalizadas, con lesiones colposcópicas extensas, utilizando anestesia regional. No hubo embarazadas sometidas a los procedimientos en esta serie.

Las muestras obtenidas fueron procesadas en la Unidad de Anatomía Patológica del Hospital Clínico Regional y Universidad de Concepción, realizando un promedio de 32 cortes histológicos por caso.

Se consideró recidiva si después de 12 meses del procedimiento las pacientes volvieron a presentar exámenes citológicos y colposcópicos positivos, y persistencia si antes de los 12 meses se presentó lesión comprobada por histología. Las pacientes que resultaron con lesiones de cáncer siguieron protocolo de acuerdo a su lesión.

Las mujeres que fueron sometidas al procedimiento recibieron la información del médico, las indicaciones, explicaciones y citación de la matrona y firmaron el consentimiento informado el día de la intervención.

Presentamos este estudio descriptivo de todas las mujeres consultantes en el período, y se analizan retrospectivamente los antecedentes obtenidos de las fichas clínicas correspondientes e ingresadas a una base con los datos en planilla electrónica. 


\section{RESULTADOS}

Del total de $1.141(37,9 \%)$ pacientes sometidas a escisión de la zona de transformación, a 1.113 $(97,5 \%)$ se les realizó el procedimiento con asa electroquirúrgica y a $28(2,5 \%)$ cono frío con bisturí.

La lesión histológica más frecuente fue la lesión intraepitelial (LIE) de alto grado con 762 casos $(66,7 \%)$, seguida por 90 casos de LIE de bajo grado (7,8\%); presentaron etapas iniciales de carcinoma invasor 74 de 1.141 mujeres $(6,5 \%)$, de este grupo la microinvasión escamosa fue la más frecuente con 36 casos $(48,6 \%)$, seguidas de lesión invasora escamosa con 22 casos $(29,7 \%)$ y 16 casos de adenocarcinoma invasor (21,6\%) (Tabla I). En 377 casos $(33 \%)$, los reportes histológicos informaron borde positivo.

Un total de 110 mujeres (9,6\%) presentaron persistencia de lesión, confirmadas por citología e histología positiva dentro del año de efectuado el procedimiento. La recidiva en esta serie fue de $0,012 \%$ con 7 casos.

El porcentaje de cura, citología y colposcopia normales, a 18 meses de seguimiento, en mujeres con LIE de alto grado fue de 85,5\% (652/762).

Tienen el antecedente de biopsia dirigida por colposcopia previa al asa electroquirúrgica 57 mujeres (5\%), dos tercios de ellas con resultados de lesiones de alto grado.

\section{DISCUSIÓN}

De las 3.004 mujeres derivadas por citología anormal, el estudio permitió descartar sin realizar biopsias, y/o tratar algunas con crioterapia, y seguimiento con controles, al mayor porcentaje de las mujeres. Este hecho es avalado por la baja sensibilidad de la citología, referida también por otros autores $(7,8)$. Un grupo pequeño fue confirmado con biopsia y tratado con histerectomía.

En las 1.141 pacientes restantes se confirmó diagnóstico de lesiones de alto grado (las más frecuentes) y también algunas lesiones invasoras iniciales. La incidencia de lesiones de alto grado $66,7 \%$ resultó similar a lo publicado en la literatura nacional $(9,10)$ y extranjera $(11)$.

Si sumamos las mujeres portadoras de LIE de bajo grado con las pacientes sometidas al procedimiento con informes histológicos sin neoplasia, se podría considerar, y tal como lo publica la literatura, como un grupo de pacientes sobretratadas y que en nuestra serie representan aproximadamente una de cada cuatro mujeres. Debemos considerar que en su doble condición de diagnóstico y tratamiento, la escisión de la zona de transformación con asa electroquirúrgica, permitió un adecuado diagnóstico de ausencia de lesión en estas últimas mujeres.

Los porcentajes reportados de la pieza histológica sin neoplasia, en citas extranjeras oscilan entre 4,5 y $25 \%(11-14)$ y de $16 \%$ en el medio nacional (9). Nuestra experiencia resulta similar con $18,4 \%$, podría eventualmente disminuir ya que hubo $5 \%$ de biopsias dirigidas previas a la escisión de la zona de transformación con asa electroquirúrgica, que podrían en algunos casos haber erradicado completamente la neoplasia.

Las cifras de LIE de bajo grado reportadas en los procedimientos con escisión de la zona de transformación con asa electroquirúrgica es muy

Tabla

\section{HISTOLOGÍA DEL CÉRVIX EN 1.141 PACIENTES SOMETIDAS A ESCISIÓN DE LA ZONA DE} TRANSFORMACIÓN: EDAD PROMEDIO Y RANGO

\begin{tabular}{lrccc}
\hline Histología & $\mathrm{n}$ & $\%$ & $\begin{array}{c}\text { Promedio } \\
\text { (años) }\end{array}$ & $\begin{array}{c}\text { Rango } \\
\text { (años) }\end{array}$ \\
\hline LIE bajo grado & 90 & 7,9 & 33,2 & $16-66$ \\
LIE alto grado & 762 & 66,8 & 34,8 & $16-75$ \\
Adenocarcinoma in situ & 5 & 0,4 & 34,4 & $29-40$ \\
Microinvasión escamosa & 36 & 3,2 & 40,2 & $24-49$ \\
Adenocarcinoma invasor & 16 & 1,4 & 41,8 & $30-62$ \\
Carcinoma escamoso invasor & 22 & 1,9 & 43,3 & $33-58$ \\
Sin Neoplasia & 210 & 18,4 & 38,6 & $17-62$ \\
\hline
\end{tabular}

LIE: lesión intraepitelial 
disímil, con reportes internacionales similares a nuestro porcentaje de $7,9 \%$ y con número inferior de casos $(11,15)$. Las cifras nacionales con casuísticas de alrededor de 140 casos oscilan entre 4,6 y $12,4 \%(9,10)$.

El porcentaje de curación, citología y colposcopia normales, con un promedio de 18 meses de seguimiento, en mujeres con LIE de alto grado fue de $85,5 \%$, similar a lo reportado internacional $(11,16,17)$ y nacionalmente $(17)$. Este buen resultado estaría relacionado con la alta frecuencia de erradicación del virus papiloma humano (HPV) después del procedimiento electro quirúrgico (18).

El bajo número de recidivas y/o persistencias confirmadas por citología e histología positiva, resultan similares a nuestras cifras, siendo menos frecuentes en LIE de bajo que las de alto grado $(14,17,19)$, también podrían explicarse por el concepto de erradicación del HPV por el procedimiento electro quirúrgico.

El informe de borde positivo de algunos de los fragmentos del cono realizado por escisión de la zona de transformación con asa electroquirúrgica y que en nuestra serie fue de $33 \%$, algo superior a lo publicado en el medio nacional (10), pero similar a lo internacional $(16,20)$. Hay que ser cuidadoso en el análisis de esta positividad, especialmente si se considera que la mayoría de estas pacientes, no presentaron anormalidades citológicas ni colposcópicas en controles de seguimiento posterior. Recientemente se ha propuesto que más que la positividad de márgenes, lo que importa como predictor de neoplasia residual es la edad y la extensión de la enfermedad medida en el porcentaje de fragmentos comprometidos, siendo significativo sobre un $40 \%$, concepto que deberíamos considerar para ser aplicado a futuros estudios (21).

Por otro lado hay que destacar que la práctica rutinaria de electrocoagulación de puntos hemorrágicos o de la totalidad del lecho cruento, incluido el borde de sección de la mucosa, debería ser interpretada como un verdadero segundo tratamiento, lo que indicaría una menor recidiva a pesar de la alta cifra de bordes comprometidos informados en el estudio histológico $(18,22)$. Por otro lado hay que considerar los reportes que presentan recidivas en escisiones completas con borde sin neoplasia, porcentajes que fluctúan entre 3 y $8 \%(20,23)$.

Las mujeres que requirieron una segunda intervención por persistencias y/o recidivas $(9,6 \%)$, como reconización e histerectomías, resulta similar a lo reportado en nuestro medio, entre 10 y $11 \%$ $(10,17)$.

El promedio de edad de las mujeres con cáncer escamoso resultó casi 10 años mayor que el promedio de edad de las LIE de alto grado $(43,5$ versus 34,8 años) concepto clásico de la historia natural de la enfermedad.

Las lesiones de carcinoma microinvasor resultaron ser 3,2\% del total de casos estudiados, mayoritariamente recibieron un segundo tratamiento.

Pensamos que cada unidad de Patología Cervical debe analizar y conocer sus propios resultados. Una casuística como la presentada requiere de varios colposcopistas, seis en nuestro caso, como asimismo varios profesionales para la citología, obtención de la muestra de histología y su análisis, lo que demuestra una dependencia del factor humano que representa una importante variable. Por otro lado, también se depende de manera sustancial de las características de la población atendida. Ambas variables se ven reflejadas en lo disímil de los resultados publicados en la literatura, de los cuáles los nuestros no son una excepción. Lo importante es conocerlos, analizarlos y enmendar los errores para optimizar los protocolos de manejo y también racionalizar el uso de los recursos.

\section{BIBLIOGRAFÍA}

1. Ministerio de Salud. Guía Clínica Cáncer Cérvicouterino. Hallado en: http://www.redsalud.gov.cl/archivos/ guiasges/CancerCervicouterino.pdf

2. Suárez E, Prieto M, Rojas I, et al. Programa Nacional de Cáncer Cérvicouterino. Rev Chil Obstet Ginecol 2001;66(6):480-91

3. Donoso E, Cuello M, Villarroel L. Reducción de la mortalidad por cáncer cérvicouterino en Chile, 1990-2003. Rev Chil Obstet Ginecol 2006;71(5):307-12.

4. van Hamont $D$, van Ham MA, Struik-van dert Zaden $\mathrm{PH}$, et al. Long-term follow up after large loop excision of the transformation zone: 22 years treatment of highgrade cervical intraepithelial neoplasia. Int J Gynecol Cancer 2006;16(2):615-9.

5. Nazzal O, Suárez E, Larraguibel R, et al. Lesiones preinvasoras de cuello uterino: una visión actual. Rev Chil Obstet Ginecol 2006;71(5):341-8.

6. Volante R, Ronco G. National survey of the quality of 2nd level in screening for cervical cancer. Epidemiol Prev 2006;30(1 suppl 3):51-7.

7. García E, Díaz J, Melo M, et al. Validación de la citología cérvico uterina convencional con prueba de referencia histopatológica en la identificación de cáncer escamocelular invasor. Rev Chil Obstet Ginecol 2006;71(3):184-8.

8. Yazigi R, Rodríguez T, Contreras L, et al. El significado clínico de dos Papanicolaou atípicos consecutivos. Rev Chil Obste Ginecol 2005;70(6):386-90.

9. Borchert G, Kusz P, Valencia O, et al. Operación cono mediante asa electroquirúrgica (LEEP) en el Hospital Regional de Copiapó: análisis del período 1994-1999, con seguimiento por 5 años hasta 2004. Rev Chil Obstet Ginecol 2006;71(4):239-45.

10. Varela J, Egaña J, González D. Conización por asa: 
experiencia en Hospital Carlos Van Buren. Rev Chil Obstet Ginecol 2002;67(1):3-9.

11. Numnum TM, Kirby TO, Leath CA 3rd, et al. A prospective evaluation of "see and treat" in women with HSIL Pap smear results: is this an appropriate strategy? J Low Genit Tract Dis 2005;9:2-6.

12. Paraskevaidis E, Koliopoulos G, Malamou-Mitsi V, et al. Large loop excision of the transformation zone for treating cervical intraepithelial neoplasia: a 12-year experience. Anticancer Res 2001;21:3097-9.

13. Howells RE, O'Mahony $\mathrm{T}$, Tucker $\mathrm{H}$, et al. How can the incidence of negative specimens resulting from large loop excision of the cervical transformation zone (LLETZ) be reduced? An analysis of the negative LLETZ specimens and development of predictive model. BJOG 2000;107(9):1075-82.

14. Ehsani N, Golbang P, Papadakis T, et al. The use of large loop excision of the transformation zone in management problems of cervical intraepithelial neoplasia. Aust N Z J Obstet Gynaecol 1998;38(3):251-3.

15. Day T, Weitzen S, Cooper AS, Boardman LA. Should unsatisfactory colposcopy necessitate treatment for cervical intraepithelial neoplasia 1? J Low Genit Tract Dis 2008;12(1):11-5.

16. Kjellberg L, Tavelin B. 'See and treat' regime by LEEP conisation is a safe and time saving procedure among women with cytological high-grade squamous intraepithelial lesion. Acta Obstet Gynecol Scand 2007;86(9):1140-4.
17. Nazzal O, Reinero M, Abarzua A, et al. Patología preinvasora del cérvix. Rev Chil Obstet Ginecol 2003;68(3):189-96.

18. Aerssens A, Claeys P, Garcia A, et al. Natural history and clearance of HPV after treatment of precancerous cervical lesions. Histopathology 2008;52(3):381-6.

19. Allam M, Paterson A, Thomson A, et al. Large loop excision and cold coagulation for management of cervical intraepithelial neoplasia. Int J Gynaecol Obstet 2005;88(1):38-43.

20. Hulman G, Pickles CJ, Gie CA, et al. Frequency of cervical intraepithelial neoplasia following large loop excision of the transformation zone. J Clin Pathol 1998;51(5):375-7.

21. Pérez Montiel D, Cantu de León D, Gómez Alvarado $\mathrm{M}$, et al. Predictors of residual disease on hysterectomy after electrosurgical excisional procedure (LEEP) with positive margins. USACAP 2008 Annual Meeting Abstracts. Poster 117. March 1-7, 2008, Denver, Colorado USA.

22. Cabrera J, Villanueva J, Correa L, et al. Uso del asa electroquirúrgica en neoplasias preclínicas del cérvix, ¿Quienes, cuantas y porqué necesitaron un segundo tratamiento? Rev Chil Obstet Ginecol 2002;67(6):4616.

23. Ghaem-Maghami S, Sagi S, Majeed G, Soutter WP. Incomplete excision of cervical intraepithelial neoplasia and risk of treatment failure: a meta-analysis. Lancet Oncol 2007;8(11):985-93. 